\title{
TGF $\beta$ (transforming growth factor $\beta$ ) superfamily members and their receptors in the fetal porcine ovaries: effect of prenatal flutamide treatment
}

\author{
Katarzyna Knapczyk-Stwora ${ }^{1}$, Malgorzata Grzesiak ${ }^{1}$, Malgorzata Duda ${ }^{1}$, \\ Marek Koziorowski ${ }^{2,3}$, Jerzy Galas ${ }^{1}$, Maria Slomczynska ${ }^{1}$ \\ ${ }^{1}$ Department of Endocrinology, Institute of Zoology, Jagiellonian University in Krakow, Poland \\ ${ }^{2}$ Department of Physiology and Reproduction of Animals, University of Rzeszow, Poland \\ ${ }^{3}$ Centre of Applied Biotechnology and Basic Sciences, University of Rzeszow, Poland
}

\begin{abstract}
Introduction. We have recently demonstrated that antiandrogen treatment during fetal life resulted in delayed folliculogenesis. The aim of the present study was to investigate the effect of androgen deficiency induced by flutamide on the expression of TGF $\beta$ superfamily members and their receptors which are involved in follicle formation and its transition to the primary stage.

Material and methods. Pregnant gilts were injected with flutamide (for seven days, $50 \mathrm{mg} / \mathrm{day} / \mathrm{kg}$ b.w.) or corn oil (controls) starting at 43 (GD50), 83 (GD90) or 101 (GD108) gestational day. The expression in fetal ovaries of selected TGF $\beta$ superfamily members (AMH, BMP4, GDF9), their receptors (AMHR-II, BMPR-IB, BMPR-II), and Smad1 and Smad3 proteins involved in signal transduction were investigated by real-time PCR and/or immunohistochemistry.

Results. Flutamide treatment increased the expression of BMP4 mRNA on GD50 and GD108 and BMPR-IB mRNA on GD50. The expression of BMPR-II was decreased at mRNA level and lower immunostaining intensity was observed after flutamide administration only on GD50. GDF9 and AMHR-II mRNA expression levels were significantly downregulated in both GD90 and GD108 groups. However, AMHR-II was immunolocalized only on GD108 and less positively stained oocytes were found after flutamide treatment. AMH mRNA level was diminished in the GD90 group, while it was elevated in the GD108 group. Moreover, the higher amounts of positively stained oocytes for phosphorylated form of Smad1 were observed following flutamide administration on GD108. Conclusions. Experimentally-induced androgen deficiency during fetal development deregulates the expression level of some of TGF $\beta$ superfamily members and their receptors which may affect primordial follicle assembly. Our findings further underline the role of androgens in the early stages of follicle development. (Folia Histochemica et Cytobiologica 2014, Vol. 52, No. 4, 317-325)
\end{abstract}

Key words: flutamide; pig; fetal ovary; AMH; BMP4; GDF9; AMHR-II; BMPR-IB; BMPR-II; qPCR, IHC

\section{Introduction}

Primordial follicles represent the ovarian reserve which has been established during fetal development (in humans) or in the very early postnatal period (in mice) [1]. Development of the follicles is tightly

Correspondence address: Prof. M. Slomczynska

Department of Endocrinology

Jagiellonian University in Krakow

Gronostajowa St. 9, 30-387 Krakow

e-mail: maria.slomczynska@uj.edu.pl regulated and many of them undergo atresia, a large number remain dormant and only a few are recruited into the growing pool $[2,3]$. There is an extensive literature describing the involvement of the members of transforming growth factor $\beta$ (TGF $\beta$ ) superfamily in each stage of follicular growth, both in fetal and postnatal life $[4,5]$. TGF $\beta$, activin, inhibin, growth differentiation factor 9 (GDF9), anti-Müllerian hormone (AMH) and several bone morphogenetic proteins (BMPs) may stimulate or inhibit paracrine communication between the oocyte, granulosa cells, and theca cells as well as autocrine signaling within 
each cell type. Moreover, there is an increasing number of evidence that locally produced growth factors play an important role in steroids production [6]. These interactions allowed controlling follicular growth and maturation.

Members of TGF $\beta$ superfamily bind to cell membrane serine/threonine kinase receptors which form a hetero-oligomeric complex of BMPR type I (BMPR-I) and BMPR type II (BMPR-II) [7]. Once the signaling cascade is initiated, the transduction of signal requires the phosphorylation of the intracellular Smad transcription factors [8]. Phosphorylation of Smads results in their ability to form complexes with other transcriptional co-factors in the nucleus and regulation of gene expression. Androgen receptor which is a ligand-activated transcription factor is tightly involved in this signaling pathway [9].

Androgens are known to play a crucial role in the female fertility, as they are essential for the follicle transition from primordial to primary stages [10,11]. Androgen receptors (ARs) are expressed in all cell types of the ovarian follicle at all stages of follicle development, including fetal life $[12,13]$. Therefore, androgen excess or deficiency is likely to have impact on the rate of follicle transition during fetal and postnatal life. Due to negligible role of gonadotropins during fetal stage of folliculogenesis, androgens are probably responsible for changes in the expression of the set of genes crucial for the development of ovarian follicles [14].

Increasing evidence suggests that primordial follicles release inhibitory factors or negative regulators which could explain why some primordial follicles remain dormant, while others are activated [15]. In the light of our earlier results which demonstrated the presence of AR in the fetal porcine ovaries [12], we assume that imbalance in the androgen milieu may affect the expression of some intraovarian factors responsible for the fetal folliculogenesis. Recently, we have demonstrated that androgen deficiency during fetal life resulted in delayed primordial and primary follicle formation [16, 17]. Moreover, we have proposed that one of the reasons of delayed folliculogenesis could be misregulation of kit ligand/ /c-Kit system [17]. Notably, the regulation of kit ligand is under control of TGF $\beta$ superfamily members [18]. Therefore, the aim of this study was to examine whether administration of an antiandrogen flutamide during mid- and late pregnancy may influence the expression of factors involved in primordial follicle formation and its transition to primary stage within developing fetal porcine ovary. To meet this goal, the expression of GDF9, AMH, BMP4, BMP receptors (BMPR-IB and BMPR-II), AMH receptor type II
(AMHR-II), and phosphorylated forms of Smad1 and Smad3 were examined using real-time PCR and immunohistochemistry.

\section{Material and methods}

Animals and experiment design. The protocol was approved by the Local Ethics Committee at the Jagiellonian University (approval no. 122/2009). Fetal ovaries used in the present investigation derived from the same experimental animals that were examined in our previous studies $[16,17]$. Briefly, sexually mature crossbred gilts $(n=12$; Large White $\times$ Polish Landrace) of similar age (10-11 months) and body weight (100-120 kg) were observed for estrus behavior and mated to fertile boars at the onset of estrus as well as 12 and $24 \mathrm{~h}$ later. The gestation day was estimated from the first mating day. Pregnant gilts were randomly allocated to three groups and injected with antiandrogen flutamide (Sigma-Aldrich, St. Louis, Missouri, USA) between days: (1) 43 and 49 of gestation (GD50, $n=2$ ), (2) 83 and 89 of gestation (GD90, $n=2$ ) and (3) 101 and 107 of gestation (GD108, $n=2)$. Flutamide was suspended in corn oil and delivered as subcutaneous injections daily for seven days at a dose of $50 \mathrm{mg} / \mathrm{kg}$ body weight. The flutamide dose was based on the results of our previous studies [19, 20], however, the time of antiandrogen administration was extended and frequency was increased. For each flutamide-exposed group, a respective control group was used $(n=2$, per each gestation period). Control animals were treated with corn oil in a manner similar to the flutamide-treated pigs. The days chosen for exposure to flutamide are critical for fetal ovarian development in pig and include the period of egg nests formation (GD50), primordial follicles formation (GD90) and their transition to primary follicles (GD108) [21].

Collection of fetal ovaries. The female fetuses were collected by hysterectomy (for details see [17]) from flutamide-treated and control pregnant gilts on day 50 of gestation $(n=9$ and $n=10$, respectively), on day 90 of gestation $(n=14$ and $n=5$, respectively) or on day 108 of gestation ( $n=11$ and $n=6$, respectively). Ovaries were immediately excised from each fetus: one ovary was snap frozen in liquid nitrogen for RNA isolation and real-time PCR analysis for AMH, AMHR-II, GDF9, BMP4, BMPR-IB, and BMPR-II while the contralateral ovary was fixed in Bouin's solution for immunohistochemical localization of AMHR-II, BMPR-II, and phosphorylated forms of Smad1 (p-Smad1) and Smad3 (p-Smad3).

Real-time PCR analysis. Total RNA was extracted from collected fetal ovaries using TRI Reagent solution (Ambion, Austin, TX, USA) following the manufacturer's instruction, resuspended in water and stored frozen at $-80^{\circ} \mathrm{C}$. The concentration of the total RNA was determined by measuring 
absorbance at 260 and $280 \mathrm{~nm}$ with a NanoDrop ND2000 Spectrophotometer (Thermo Scientific, Wilmington, DE, USA). Moreover, RNA samples were electrophorezed on a $1 \%(\mathrm{w} / \mathrm{v})$ denaturing agarose gel to verify the RNA quality. A reverse transcription was carried out using $1 \mu \mathrm{g}$ of total RNA and a High-Capacity cDNA Reverse Transcription kit (Applied Biosystems, Carlsbad, CA, USA) following the manufacturer's protocol. In brief, a reaction mixture was prepared in $20 \mu \mathrm{L}$ volume using random primers, dNTP mix, RNAse inhibitor and Multi Scribe Reverse Transcriptase.

Reverse transcription was performed in Veriti Thermal Cycler (Applied Biosystems), using the following parameter values: $25^{\circ} \mathrm{C}$ for $10 \mathrm{~min}, 37^{\circ} \mathrm{C}$ for $120 \mathrm{~min}$, and $85^{\circ} \mathrm{C}$ for 5 min. Genomic DNA contamination was checked by control experiments in which reverse transcriptase was omitted during the RT step. Samples were kept at $-20^{\circ} \mathrm{C}$ until further analysis.

Real time PCR analyses were performed using the StepOne ${ }^{\mathrm{TM}}$ Real-Time PCR System (Applied Biosystems) according to the recommended cycling program $\left(2 \mathrm{~min}\right.$ at $50^{\circ} \mathrm{C}$, 10 min at $95^{\circ} \mathrm{C}, 40$ cycles of $15 \mathrm{sec}$ at $95^{\circ} \mathrm{C}$, and $1 \mathrm{~min}$ at $60^{\circ} \mathrm{C}$ ). The expression level of the $A M H, A M H R-I I, G D F 9, B M P 4$, $B M P R-I B$ and $B M P R-I I$ was quantified in each sample utilizing TaqMan Gene Expression Assays (Ss03383931_u1 for $A M H$, Ss04321772_m1 for AMHR-II, Ss03391680_m1 for GDF9, Ss03386419_u1 for BMP4, Ss03380019_u1 for BMPR-IB, and Ss04248590_m1 for BMPR-II; Applied Biosystems). GAPDH levels were used as endogenous control (assay ID: Ss03373286_u1). Real-time PCR reactions were performed in $20 \mu \mathrm{L}$ of total reaction volume which contained $10 \mu \mathrm{L}$ of TaqMan Gene Expression Master Mix (Applied Biosystems), $1 \mu \mathrm{L}$ of TaqMan Gene Expression Assay, $8 \mu \mathrm{L}$ of water, and $2 \mu \mathrm{L}$ of analyzed sample.

Expression levels of investigated genes were normalized to GAPDH expression and determined using $2^{-\Delta \mathrm{Ct}}$ method [22]. Briefly, the cycle threshold $(\mathrm{Ct})$ of $G A P D H$ (defined as the cycle number at which the fluorescence exceeds the threshold level) was subtracted from the $\mathrm{Ct}$ value of the gene of interest $(\Delta \mathrm{Ct})$ and relative expression was presented as $2^{-\Delta \mathrm{Ct}}$.

Immunohistochemistry. Fixed and embedded in paraplast (Sigma-Aldrich) fetal ovaries were cut into $5 \mu \mathrm{m}$ sections and mounted onto 3'3'-aminopropyl-triethoxysaline (APES, Sigma-Aldrich) coated slides. Then sections were dewaxed, rehydrated and rinsed in water. Microwave antigen retrieval was performed in $0.01 \mathrm{M}$ citrate buffer, $\mathrm{pH} 6.0(3 \times 4 \mathrm{~min}$, $750 \mathrm{~W})$. Endogenous peroxidase activity was quenched by 20 min incubation of slides with $0.3 \% \mathrm{H}_{2} \mathrm{O}_{2}$ in TBS (Tris-buffered saline, $\mathrm{pH}$ 7.4). Blocking of non-specific binding sites was performed with $5 \%(\mathrm{v} / \mathrm{v})$ normal goat serum (for p-Smad1, p-Smad3 and BMPR-II), or $10 \%(\mathrm{v} / \mathrm{v})$ normal goat serum (for AMHR-II) prior to incubation with the primary antibodies. The following rabbit polyclonal antibodies and dilutions were applied: anti-p-Smad1 (1:200,
Santa Cruz Biotechnology Inc., Santa Cruz, CA, USA, sc-12353R), anti-p-Smad3 (1:2000, Abcam, ab-51451), anti -AMHR-II (1:50, Santa Cruz Biotechnology Inc., sc-67287), and anti-BMPR-II (1:100, Santa Cruz Biotechnology Inc., sc-20737). After overnight incubation at $4^{\circ} \mathrm{C}$ in a humidified chamber, the antigens were visualized using biotinylated goat anti-rabbit secondary antibody (1:300, $1.5 \mathrm{~h}$ at room temperature; Vector Laboratories, Burlingame CA, USA), avidin-biotin-peroxidase complex (40 min at room temperature; StreptABComplex-HRP, Vector Laboratories), and 3,3'-diaminobenzidine (Sigma-Aldrich) as a chromogen. Counterstaining was performed with Mayer's hematoxylin for BMPR-II. For the negative control reaction, sections were incubated with non-immune rabbit $\operatorname{IgG}$ instead of primary antibodies and processed as above. Selected sections were photographed using a Nikon Eclipse Ni-U microscope and a Nikon Digital DS-Fi1-U3 camera (Nikon, Tokyo, Japan) with corresponding software.

Statistical analysis. Statistical analyses were performed using Statistica v. 10 program (StatSoft, Inc., Tulsa, OK, USA). All data are presented as the overall mean \pm SEM Normal distribution of data was tested with the Shapiro -Wilk test. Then, the nonparametric Mann-Whitney $U$-test was used to determine significant differences between the control and flutamide-treated groups. Data were considered to be statistically significant at the $95 \%$ confidence level $(p<0.05)$.

\section{Results}

\section{Effect of flutamide on AMH, AMHR-II, GDF9, BMP4, BMPR-IB and BMPR-II IRNA expression in fetal ovaries}

Due to the lack of ovarian follicles on day 50 of gestation the expression of AMH, AMHR-II and GDF9 was not examined in the GD50 group. On GD50, real -time PCR analysis revealed that flutamide treatment caused upregulation of BMP4 ( $p<0.001$, Figure 1D) and $B M P R-I B(p<0.01$, Figure $1 \mathrm{E})$ expression and downregulation of BMPR-II ( $p<0.001$, Figure 1F) expression. On GD90, flutamide administration resulted in a decreased level of AMH $(p<0.01)$, AMHR -II $(p<0.05)$ and GDF9 $(p<0.01)$ mRNAs levels (Figure $1 \mathrm{~A}-\mathrm{C}$, respectively), while no changes were observed in BMP4, BMPR-IB and BMPR-II expression (Figure1D-F).On GD108, the level of AMH(Figure1A) and BMP4 (Figure 1D) mRNAs was significantly higher ( $p<0.01, p<0.05$, respectively) in flutamide-treated group, however, the level of AMHR-II (Figure 1B) and GDF9 (Figure 1C) mRNAs was lower $(p<0.01)$ when compared to the control group. The expression of BMPR-IB and BMPR-II genes was not significantly 
A

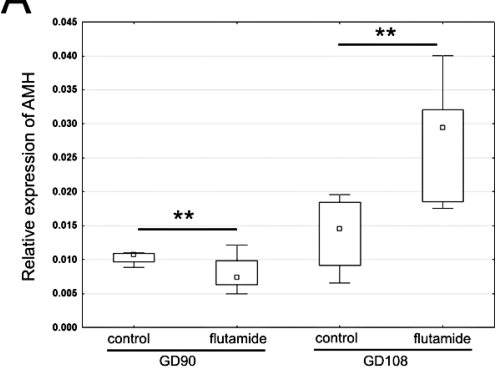

D

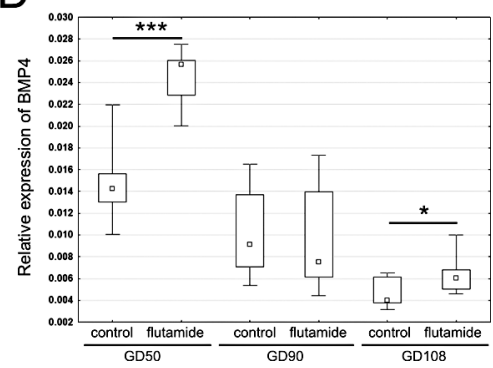

B

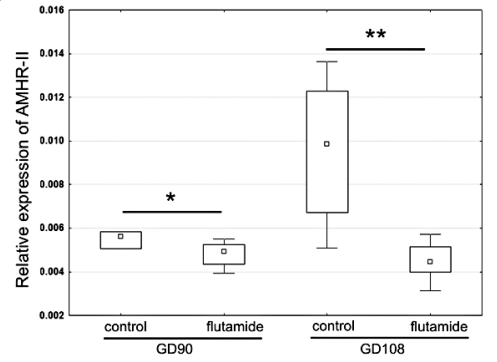

E

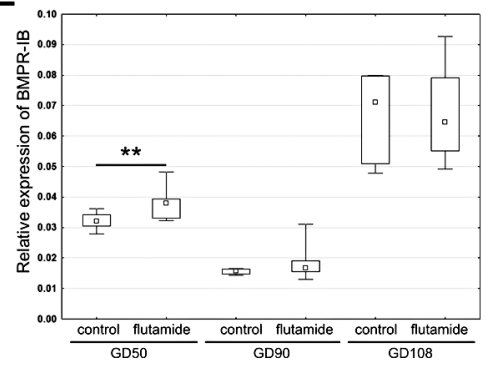

C

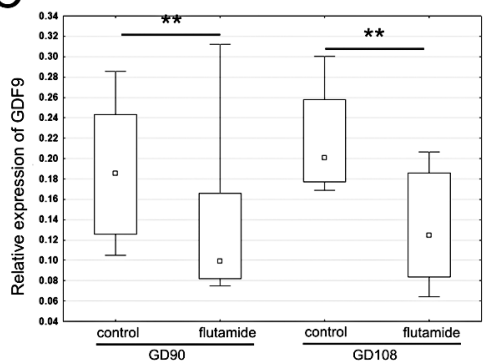

$\mathrm{F}$

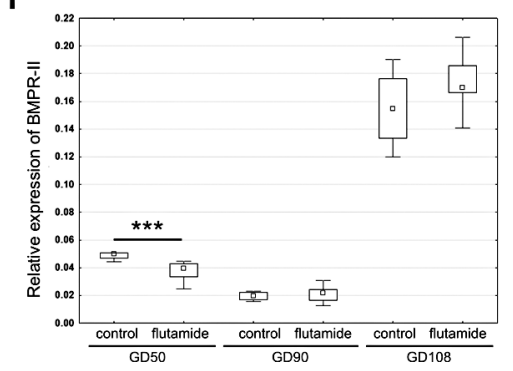

Figure 1. Relative gene expression of TGF $\beta$ superfamily members and their receptors in the fetal porcine ovaries. Relative levels of AMH (A), AMHR-II (B), GDF9 (C), BMP4 (D), BMPR-IB (E) and BMPR-II (F) mRNAs in porcine ovaries obtained from control and flutamide-exposed fetuses on days 50 (GD50), 90 (GD90) and 108 (GD108) of gestation were determined using quantitative real-time PCR analysis. Data are presented as $2^{-\Delta \mathrm{Ct}}$ relative to GAPDH expression. The box plot shows medians (dot within the boxes) and the 25-75 percentiles (box sizes), and whiskers indicate minimum and maximum. Because of the lack of statistical normality, $P$ values were calculated with the Mann-Whitney $U$-test. Asterisks denote statistically significant differences $\left({ }^{*} p<0.05,{ }^{* *} p<0.01,{ }^{* * *} p<0.001\right)$
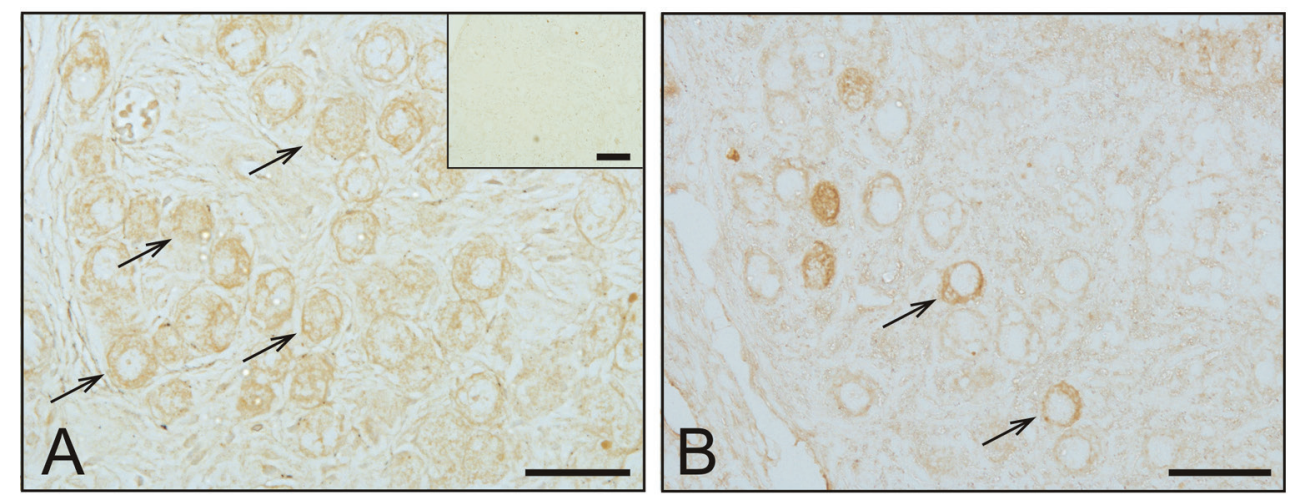

Figure 2. AMHR-II immunostaining in the fetal porcine ovaries. Ovaries were obtained from control (A) and flutamide-exposed (B) fetuses on day 108 of gestation. Arrows indicate positive AMHR-II staining in the oocytes of primary ovarian follicles from both control and flutamide-exposed fetuses. Control sections, in which the primary antibody was replaced by rabbit IgG, did not exhibit any positive staining (A, inset). All the scale bars represent $50 \mu \mathrm{m}$

altered by flutamide treatment in the GD108 group (Figure 1E, F).

\section{Effect of flutamide on AMHR-II, BMPR-II, p-Smad1 and p-Smad3 localization in fetal ovaries}

AMHR-II immunostaining was detected in the ovaries obtained from control and flutamide-exposed fe- tuses only in the GD108 group (Figure 2A, B, respectively). AMHR-II immunoreactivity was present in the oocytes of primary ovarian follicles. After flutamide treatment, less AMHR-II positive oocytes was found as compared with the control group (Figure 2A, B).

In all examined sections, immunoreactive BMPR-II antigens were expressed in the stromal cells of the ovaries obtained from control and flutamide-treated 

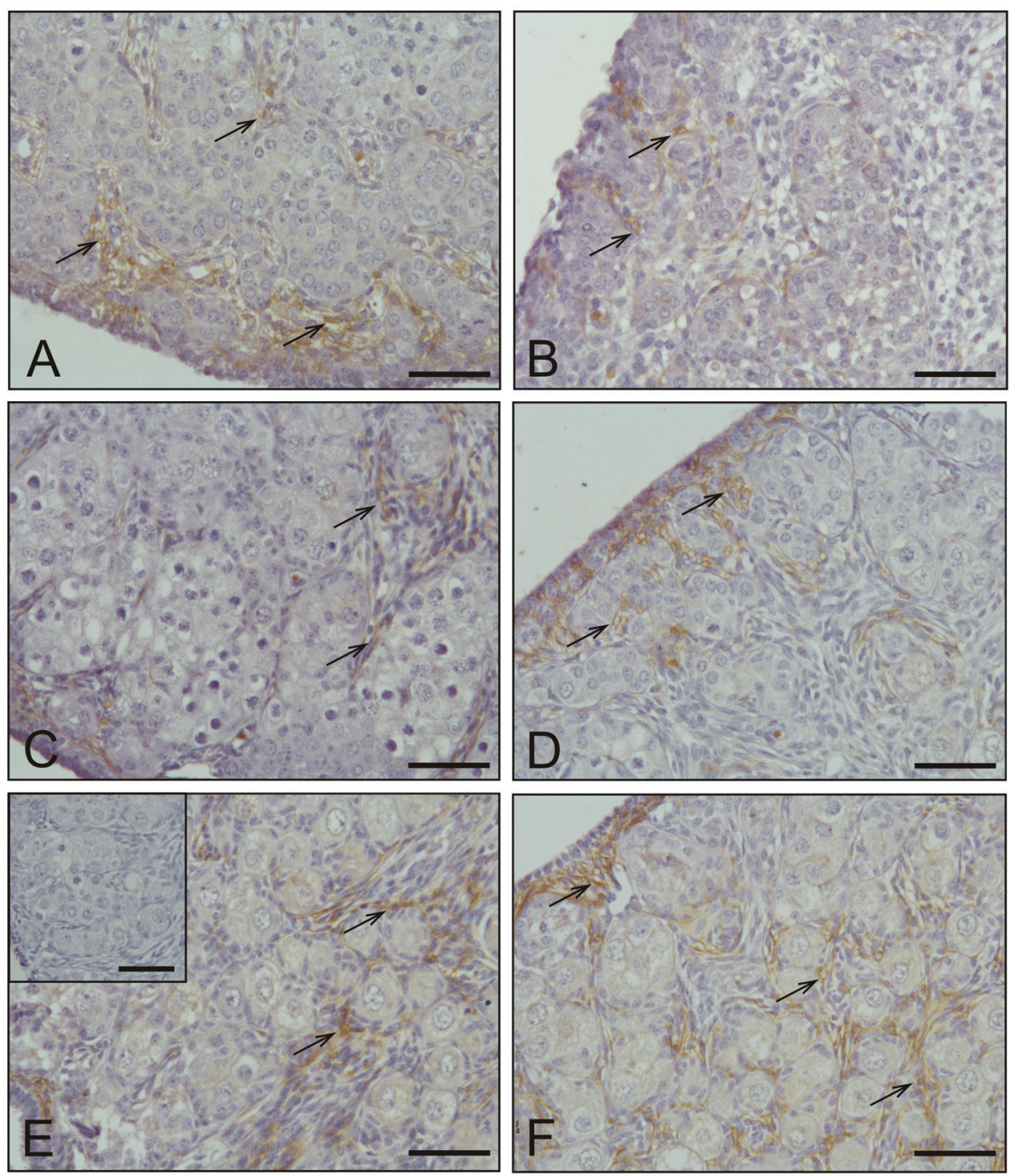

Figure 3. BMPR-II immunostaining in the fetal porcine ovaries. Ovaries were obtained on days 50 (A, B), 90 (C, D) and 108 (E, F) of gestation from control (A, C, E) and flutamide-exposed (B, D, F) fetuses. In all representative micrographs, arrows indicate positive BMPR-II staining in the stromal cells from both control and flutamide-exposed fetuses. Control sections, in which the primary antibody was replaced by rabbit $\mathrm{IgG}$, did not exhibit any positive staining (E, inset). All the scale bars represent $50 \mu \mathrm{m}$

fetuses on GD50 (Figure 3A, B, respectively), GD90 (Figure 3C, D, respectively) and GD108 (Figure 3E, F, respectively). On GD50, flutamide treatment resulted in lower intensity of BMPR-II immunostaining as compared with the control group (Figure 3A, B). Flutamide administration did not affect the intensity of BMPR-II immunoreactivity in the GD90 and GD108 groups.

Positive p-Smad1 nuclear staining was observed in fetal ovaries from control and flutamide-treated fetuses on GD90 (Figure 4A, B, respectively) and GD108 (Figure 4C, D, respectively). Fetal ovaries in the GD50 group were immunonegative for p-Smad1. Immunohistochemistry revealed p-Smad1 localization in the oocytes within nests. Flutamide treatment did not affect the intensity of p-Smad1 immunostaining, and more p-Smad1 positive oocytes were found on GD108 as compared with the control group.

Immunohistochemistry for $\mathrm{p}$-Smad3 revealed nuclear staining in ovarian surface epithelium and oogonia/oocytes nests in the fetal ovaries obtained from control and flutamide-treated fetuses on GD50 (Figure 5A, B, respectively), GD90 (Figure 5C, D, respectively) and GD108 (Figure 5E, F, respectively). p-Smad3 stromal immunopositive cells were found on GD50 (Figure 5A, B) and GD90 (Figure 5C, D), while on GD108, p-Smad3 was demonstrated in granulosa 

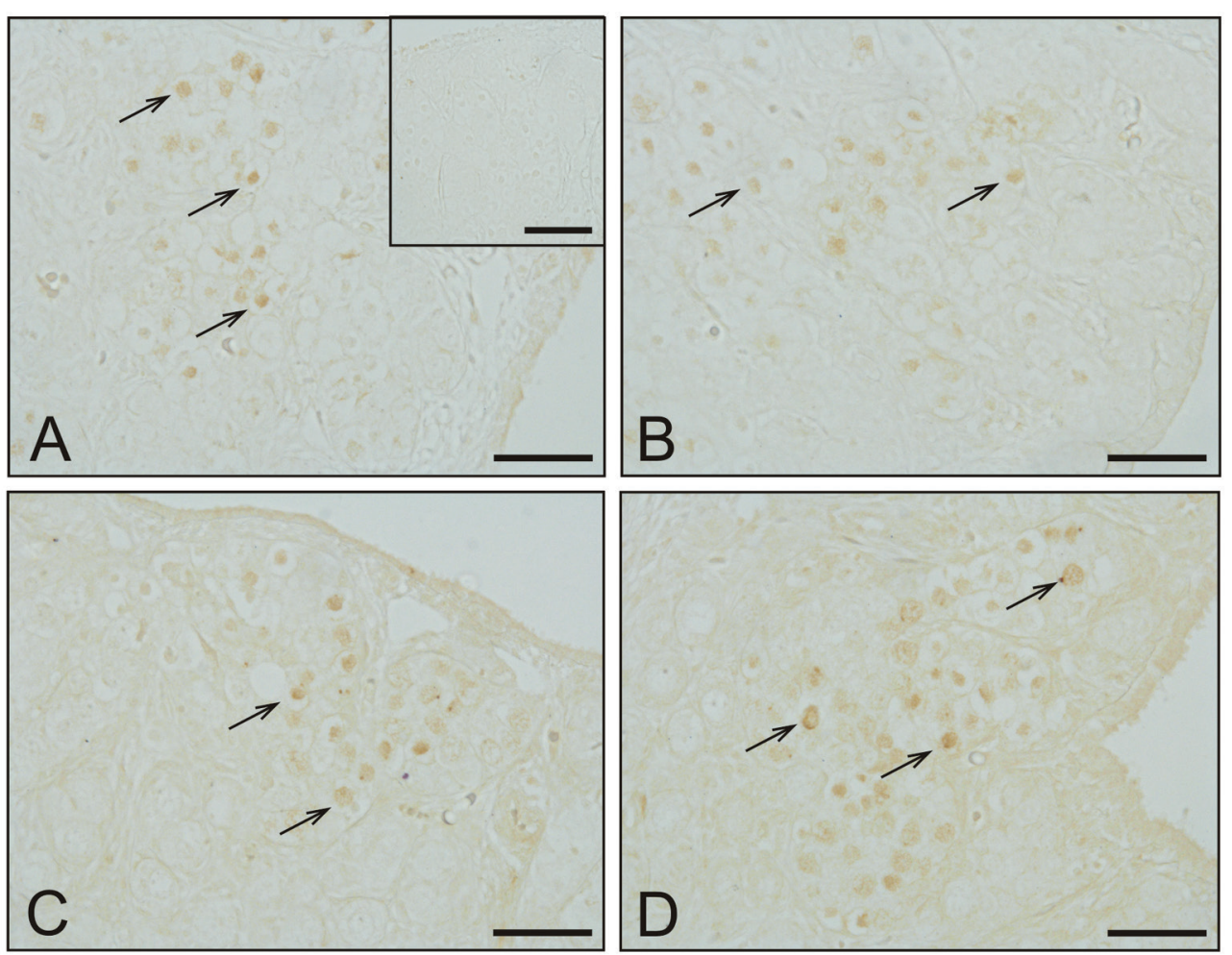

Figure 4. Immunolocalization of phosphorylated form of Smad1 (p-Smad1) in the fetal ovaries. Ovaries were obtained on days 90 (A, B) and 108 (C, D) of gestation from control (A, C) and flutamide-exposed (B, D) fetuses. Arrows point at oocytes' nuclei showing a positive p-Smad1 immunostaining in both control and flutamide-exposed fetuses (A-D). Control section, in which the primary antibody against p-Smad1 was replaced by rabbit IgG, did not exhibit any positive staining (A, inset). All the scale bars represent $50 \mu \mathrm{m}$

cells of forming follicles (Figure 5E, F). Flutamide administration did not affect the intensity of p-Smad3 immunostaining in all examined experimental groups as compared with their respective control groups.

\section{Discussion}

Porcine ovarian development is distinct from that of the other animals as both follicle formation and its transition to the primary stage occur during fetal life [21]. Previously, we have found that disruption of androgen signaling during critical periods of porcine folliculogenesis may delay primordial follicle formation and its transition to the primary stage by affecting the expression of many genes involved in the early stages of folliculogenesis $[16,17]$. Among them, the kit ligand/c-kit signaling system, which can be affected by androgens, was shown to be relevant to the initiation of follicular transition from primordial into primary follicles $[17,23]$. Notably, the regulation of kit ligand is under control of TGF $\beta$ superfamily [18]. Therefore, the further step toward the understanding the role of androgens in the early stages of folliculogenesis is to address the question whether androgen deficiency disturbs the expression of BMP4, GDF9, AMH and their receptors in the fetal porcine ovary.

It was shown that the fetal porcine ovaries expressed BMPRs from the day 46 of gestation in oogonia and oocytes within egg nests, which suggests the involvement of BMP signaling in oogonial proliferation and follicle formation [24]. Among numerous BMPs, BMP4 has been shown to promote oocyte survival and the primordial to primary follicle transition. Ovaries from 4-day-old rats, treated in vitro with BMP4, revealed a higher proportion of developing primary follicles and a reduced number of primordial follicles [25]. In the current study, we showed that treatment with an antiandrogen resulted in the differential expression of BMP4 mRNA in the porcine fetal ovaries. Moreover, we have demonstrated that the expression of BMPR-IB and BMPR-II genes is controlled by flutamide. It is possible, therefore, that changed BMP signaling resulting from antiandrogen treatment may affect the recruitment of pre-granulosa cells around the oocyte and follicle activation. However, the sensitivity of ovarian compartments to exogenous antiandrogens may vary within fetal development which was demonstrated previously [16]. 

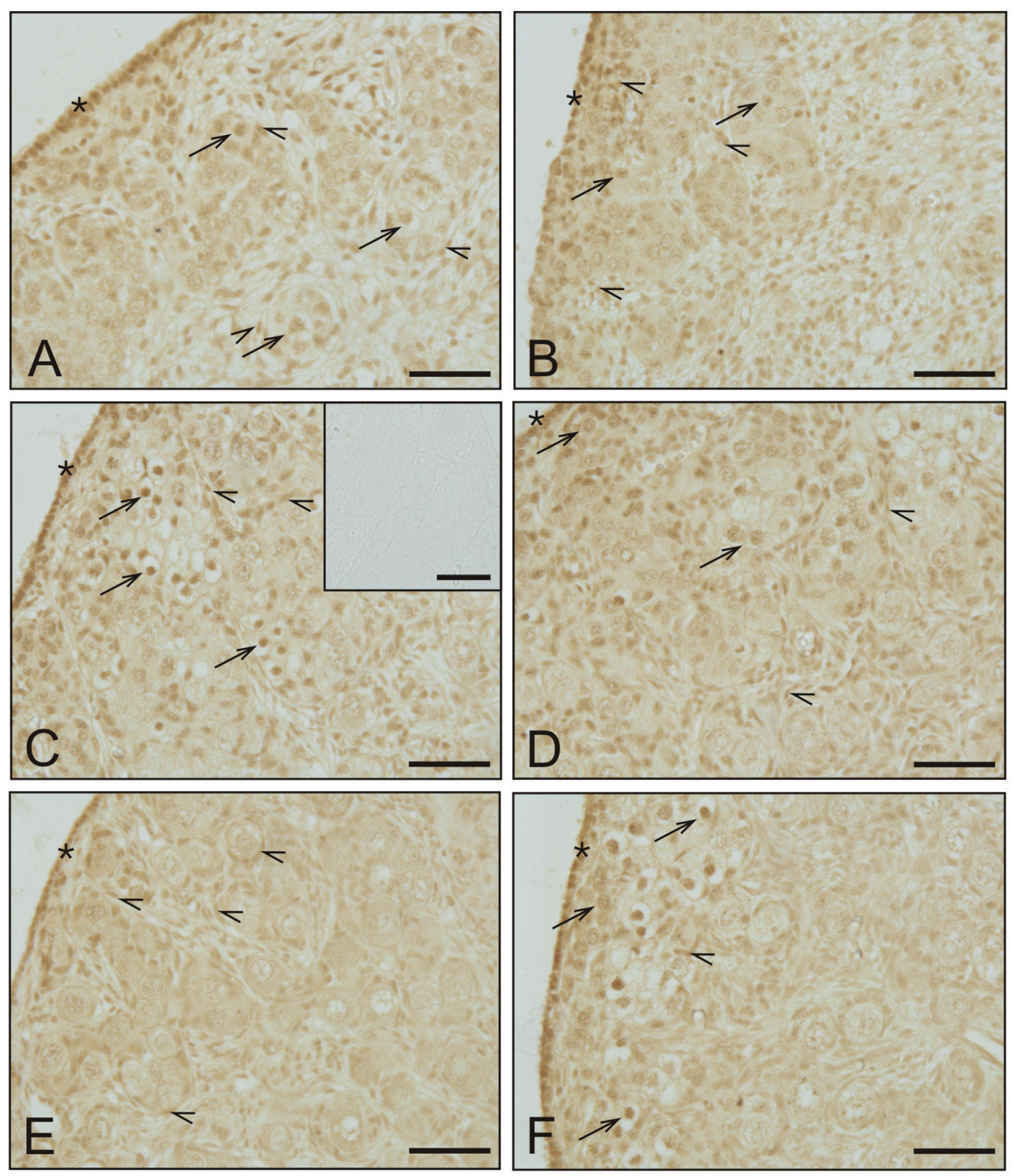

Figure 5. Immunolocalization of phosphorylated form of Smad3 (p-Smad3) in the fetal ovaries. Ovaries were obtained on days 50 (A, B), 90 (C, D) and 108 (E, F) of gestation from control (A, C, E) and flutamide-exposed (B, D, F) fetuses. Nuclear positive staining for $\mathrm{p}$-Smad3 was found in ovarian surface epithelium (asterisks) and within egg nests (arrows) from both control and flutamide-exposed fetuses. On days 50 and 90 of gestation open arrowheads indicate positive p-Smad3 staining in the stromal cells (A-D), while on day 108 of gestation open arrowheads indicate positive p-Smad3 staining in the granulosa cells of forming follicles $(\mathrm{E}, \mathrm{F})$. Control sections, in which the primary antibody was replaced by rabbit IgG, did not exhibit any positive staining $(\mathrm{C}$, inset). All the scale bars represent $50 \mu \mathrm{m}$

The signal transduction of the TGF $\beta$ superfamily is mediated by Smad proteins. The BMPs group largely signals via Smad1, Smad5, and Smad8 [26]. Among other functions, Smads are known to play a role of transcriptional co-modulators, which can positively or negatively regulate activation of target genes. Once induced by BMPs, phosphorylated Smad1 physically interacts with AR and suppresses its function [9]. It was also reported that AR associates with Smad3 resulting in either activation [27] or inhibition [28] of
AR-mediated transcription. In the present study, the phosphorylated form of Smad1 was observed in the oocytes within egg nests in the fetal ovaries during late gestational period. Flutamide treatment did not affect the intensity of immunostaining but higher number of positively stained oocytes were observed on GD108. On the other hand, phosphorylated form of Smad3 (p-Smad3) was identified in ovarian surface epithelium and oogonia/oocytes nests regardless the investigated day of gestation. In addition, it was observed 
in stromal cells earlier in development, while later it was demonstrated in granulosa cells of the forming follicles. However, flutamide administration did not affect the intensity of p-Smad3 staining in all examined groups. Results presented herein may suggest participation of BMP/Smad1 signaling in response to androgen withdrawal. Due to a possible association of Smad1 with AR [9], the observed changes in p-Smad1 immunolocalization following antiandrogen administration may influence androgen-regulated gene expression. On the other hand, the lack of changes in the Smad3 phosphorylation following flutamide administration may be caused by the other factors underlying Smad3 regulation such as the activity of protein phosphatases [29].

GDF9 appears to be a general marker for oocyte and follicle health status, and is expressed exclusively in the oocyte throughout the entire period of follicle development [30]. Targeted deletion of GDF9 in the mouse revealed its essential role in folliculogenesis. It was reported that GDF9-deficient female mice are infertile with a well-described follicular block at the primary one-layer follicle stage which leads to complete infertility [31]. However, Vitt and Hsueh [30] suggested that GDF9 is not absolutely required for the transition from primordial to primary follicles, but stimulates primary follicle progression. Thus, oocyte-derived GDF9 was shown to be required for ovarian somatic cell function [30]. A widely accepted opinion is that GDF9 mediates its signal via BMPR-II, but its downstream actions are mediated by activation of the $\operatorname{Smad} 2$ and $\mathrm{Smad} 3$ proteins $[32,33]$. Due to the lack of ovarian follicles on day 50 of gestation, we examined GDF9 expression only during late pregnancy. Our finding that flutamide administration resulted in a decreased level of GDF9 mRNA expression the GD90 and GD108 groups confirms our previous notion that androgen deficiency during late gestational period led to delayed folliculogenesis [17] and the observed diminished expression of GDF9 could be at least partly responsible for these phenomena. However, neither BMPR-II expression nor Smad3 phosphorylation was diminished following flutamide treatment. Thus, other factors may be involved in BMPR-II and Smad3 activation.

In the ovarian follicles, $\mathrm{AMH}$ is produced by granulosa cells from the primary to the early antral follicle stage, at which its expression is suppressed by FSH [34]. The decrease in AMH level leads to premature primordial follicle activation, as AMH inhibits primordial to primary follicle transition acting as a modifier of the rate of follicle assembly [35]. In the current study, the antiandrogen treatment resulted in the diminished expression of AMH mRNA level in the GD90 group, while it was elevated in the GD108 group. These results may indicate that in pigs inhibitory action of AMH is most likely exerted around parturition period, and the higher level of AMH following flutamide treatment may lead to the maintenance of primordial follicles during this period. However, AMHR-II was immunolocalized only on GD108. Moreover, flutamide administration decreased its expression at mRNA level as well as less AMHR-II positive oocytes was found as compared with the control group on GD108. In vitro studies demonstrated that AMH signals may also be transduced through ALK2, the type of BMPR-I, and Smad1 pathway [36]. Therefore, we assume that in spite of the decreased AMHR-II expression on GD108, AMH may still exert its inhibiting role.

The members of TGF $\beta$ superfamily act as a stage- and cell-specific paracrine factors that, together with the steroid hormones, orchestrate the timely growth and development of the follicle. In the present study, experimentally-induced androgen deficiency during fetal development deregulated the level of some of the TGF $\beta$ superfamily members and its receptors which may affect primordial follicle assembly. Notably, our recent studies demonstrated that the restricted action of androgens induced by flutamide administration during mid- and late pregnancy resulted in delayed primordial follicle formation and its transition to the primary stage $[16,17]$. Therefore, we propose that an interaction between factors secreted by oocyte/granulosa cells and androgens could regulate primordial to primary follicle transition. The presented findings further underline the role of androgens in the early stages of follicle development.

\section{Acknowledgments}

This work was partially supported by the Ministry of Science and Higher Education, a grant No. N N303 596539 (to M.S.) and the Jagiellonian University grant K/ZDS/0004192.

\section{References}

1. Peters H, Byskov AG, Himelstein-Braw R, Faber M. Follicular growth: the basic event in the mouse and human ovary. J Reprod Fertil. 1975;45:559-566.

2. Tingen C, Kim A, Woodruff TK. The primordial pool of follicles and nest breakdown in mammalian ovaries. Mol Hum Reprod. 2009;15:795-803.

3. Kim JY. Control of ovarian primordial follicle activation. Clin Exp Reprod Med. 2012;39:10-14.

4. Findlay JK, Drummond AE, Dyson ML, Baillie AJ, Robertson DM, Ethier JF. Recruitment and development of the follicle; the roles of the transforming growth factor-beta superfamily. Mol Cell Endocrinol. 2002;191:35-43. 
5. Knight PG, Glister C. Local roles of TGF-beta superfamily members in the control of ovarian follicle development. Anim Reprod Sci. 2003;78:165-183.

6. Ruutiainen K, Adashi EY. Intraovarian factors in hyperandrogenism. Semin Reprod Endocrinol. 1993;11:324-328.

7. Massagué J. TGF-beta signal transduction. Annu Rev Biochem. 1998;67:753-791.

8. Hill CS. Nucleocytoplasmic shuttling of Smad proteins. Cell Res. 2009;19:36-46.

9. Qiu T, Grizzle WE, Oelschlager K, Shen X, Cao X. Control of prostate cell growth: BMP antagonizes androgen mitogenic activity with incorporation of MAPK signals in Smad1. EMBO J. 2007;26:346-357.

10. Drummond AE. The role of steroids in follicular growth. Reprod Biol Endocrinol. 2006;4:16.

11. Walters KA, Allan CM, Handelsman DJ. Androgen actions and the ovary. Biol Reprod. 2008;78:380-389.

12. Burek M, Duda M, Knapczyk K, Koziorowski M, Słomczyńska $\mathrm{M}$. Tissue-specific distribution of the androgen receptor (AR) in the porcine fetus. Acta Histochem. 2007;109:358-365.

13. Sen A, Hammes SR. Granulosa cell-specific androgen receptors are critical regulators of ovarian development and function. Mol Endocrinol. 2010;24:1393-1403.

14. Prizant H, Gleicher N, Sen A. Androgen actions in the ovary: balance is key. $J$ Endocrinol. 2014;222:R141-R151.

15. Da Silva-Buttkus P, Marcelli G, Franks S, Stark J, Hardy K. Inferring biological mechanisms from spatial analysis: prediction of a local inhibitor in the ovary. Proc Natl Acad Sci USA. 2009;106:456-461.

16. Knapczyk-Stwora K, Durlej-Grzesiak M, Ciereszko RE, Koziorowski M, Slomczynska M. Antiandrogen flutamide affects folliculogenesis during fetal development in pigs. Reproduction. 2013;145:265-276.

17. Knapczyk-Stwora K, Grzesiak M, Duda M, Koziorowski M, Slomczynska M. Effect of flutamide on folliculogenesis in the fetal porcine ovary - regulation by Kit Ligand/c-Kit and IGF1/IGF1R systems. Anim Reprod Sci. 2013;142:160-167.

18. Pangas SA. Regulation of ovarian reserve be members of the transforming growth factor beta family. Mol Reprod Dev. 2012;79:666-679.

19. Durlej M, Kopera I, Knapczyk-Stwora K et al. Connexin43 gene expression in male and female gonads of porcine offspring following in utero exposure to an anti-androgen, flutamide. Acta Histochem. 2011;113:6-12.

20. Durlej M, Knapczyk-Stwora K, Slomczynska M. Prenatal and neonatal flutamide administration increases proliferation and reduces apoptosis in large antral follicles of adult pigs. Anim Reprod Sci. 2012;132:58-65.

21. Bielanska-Osuchowska Z. Oogenesis in pig ovaries during prenatal period: ultrastructure and morphometry. Reprod Biol. 2006;6:161-193.
22. Livak KJ, Schmittgen TD. Analysis of relative gene expression data using real-time quantitative PCR and the $2^{-\Delta \Delta C t}$ method. Methods. 2001;25:402-408.

23. Shiina H, Matsumoto T, Sato T et al. Premature ovarian failure in androgen receptor-deficient mice. Proc Natl Acad Sci USA. 2006;103:224-229.

24. Quinn RL, Shuttleworth G, Hunter MG. Immunohistochemical localization of the bone morphogenetic protein receptors in the porcine ovary. J Anat. 2004;205:15-23.

25. Nilsson EE, Skinner MK. Bone morphogenetic protein- 4 acts as an ovarian follicle survival factor and promotes primordial follicle development. Biol Reprod. 2003;69:1265-1272.

26. Gong X, McGee EA. Smad3 is required for normal follicular follicle-stimulating hormone responsiveness in the mouse. Biol Reprod. 2009;81:730-738.

27. Kang HY, Lin HK, Hu YC, Yeh S, Huang KE, Chang C. From transforming growth factor-beta signaling to androgen action: identification of $\mathrm{Smad} 3$ as an androgen receptor coregulator in prostate cancer cells. Proc Natl Acad Sci USA. 2001;98:3018-3023.

28. Hayes SA, Zarnegar M, Sharma M et al. SMAD3 represses androgen receptor-mediated transcription. Cancer Res. 2001;61:2112-2118.

29. Lin X, Chen Y, Meng A, Feng X. Termination of TGF-beta superfamily signaling through SMAD dephosphorylation a functional genomic view. J Genet Genomics. 2007;34:1-9.

30. Vitt UA, Hsueh AJ. Stage-dependent role of growth differentiation factor-9 in ovarian follicle development. Mol Cell Endocrinol. 2001;183:171-177.

31. DongJ,AlbertiniDF,NishimoriK,KumarTR,LuN,MatzukMM. Growth differentiation factor-9 is required during early ovarian folliculogenesis. Nature. 1996;383:531-535.

32. Vitt UA, Mazerbourg S, Klein C, Hsueh AJ. Bone morphogenetic protein receptor type II is a receptor for growth differentiation factor-9. Biol Reprod. 2002;67:473-480.

33. Kaivo-Oja N, Bondestam J, Kämäräinen $\mathrm{M}$ et al. Growth differentiation factor-9 induces $\mathrm{Smad} 2$ activation and inhibin $\mathrm{B}$ production in cultured human granulosa-luteal cells. J Clin Endocrinol Metab. 2003;88:755-762.

34. Durlinger AL, Gruijters MJ, Kramer P et al. Anti-Müllerian hormone attenuates the effects of FSH on follicle development in the mouse ovary. Endocrinology. 2001;142: 4891-4899.

35. Durlinger AL, Gruijters MJ, Kramer P et al. Anti-Müllerian hormone inhibits initiation of primordial follicle growth in the mouse ovary. Endocrinology. 2002;143:1076-1084.

36. Clarke TR, Hoshiya Y, Yi SE, Liu X, Lyons KM, Donahoe PK. Müllerian inhibiting substance signaling uses a bone morphogenetic protein (BMP)-like pathway mediated by ALK2 and induces SMAD6 expression. Mol Endocrinol. 2001;15:946-959. 